\title{
Trastornos de la alimentación en pacientes hospitalizados en un Servicio de Salud Mental Pediátrico
}

\author{
Eating behavior disorders in patients hospitalized in a Mental Health Service
}

\author{
Andrea Corral $^{\mathrm{a}}$, Valeria Espinoza ${ }^{\mathrm{a}}$, Karla Yohannessen ${ }^{\mathrm{b}, \mathrm{c}}$, \\ Paula Loyola $^{\mathrm{d}}$, Paulina Balboa ${ }^{\mathrm{c}, \mathrm{e}}$, Claudia Torrejón ${ }^{\mathrm{c}, e}$
}

\author{
aBecaria de Pediatría, Departamento de Pediatría y Cirugía Infantil, Campus Norte, Universidad de Chile \\ bEscuela de Salud Pública, Universidad de Chile \\ 'Departamento de Pediatría y Cirugía Infantil, Campus Norte. Universidad de Chile \\ dHospital Clínico Magallanes, Punta Arenas \\ eUnidad de Nutrición Infantil, Hospital Roberto del Río, Santiago, Chile
}

Recibido: 3 de julio de 2018; Aprobado: 3 de enero de 2019

\section{Resumen}

Los trastornos de la alimentación e ingesta (TAI) tienen una elevada prevalencia durante la adolescencia, asociándose a alta morbimortalidad. En nuestro país no existen datos que caractericen a los adolescentes con TAI que requieren hospitalización. Objetivo: Describir y analizar las hospitalizaciones debidas a TAI en niños y adolescentes en un Servicio de Salud Mental Pediátrico (SSMP). Pacientes y Método: Se recolectaron los datos de los registros clínicos de pacientes con TAI hospitalizados en el SSMP del Hospital Roberto del Río entre 2005-2015. Se estudiaron las variables de ingreso: motivo de hospitalización, tipo de TAI, estado nutricional, repercusión sistémica y variables sicosociales (comorbilidades siquiátricas, funcionamiento de la familia, abuso e ideación/intento suicida). Para la comparación de variables cuantitativas entre grupos se utilizó el test t-Student y para variables categóricas chi-cuadrado o Test Fisher. Resultados: Se incluyeron 93 pacientes, mediana de edad 14,6 años, $84 \%$ género femenino. El diagnóstico más frecuente fue anorexia nerviosa (AN) (71\%) y la causa más frecuente de hospitalización fue el fracaso del tratamiento ambulatorio, seguido por ideación/intento suicida. $\mathrm{Al}$ ingreso, $40 \%$ de los pacientes presentaban déficit nutricional, 96\% comorbilidad psiquiátrica y $88 \%$ disfunción familiar. Conclusión: La AN fue el TAI más frecuente dentro de los pacientes hospitalizados y el fracaso del tratamiento ambulatorio el principal motivo de ingreso. Esto último podría ser explicado, en parte, por la alta prevalencia de disfunción familiar y comorbilidad psiquiátrica de los pacientes y su familia, que estaría complicando el tratamiento ambulatorio.
Palabras clave:

Trastornos de conducta alimentaria; anorexia nerviosa; adolescentes hospitalizados; funcionamiento familiar 


\begin{abstract}
Eating disorders (ED) have a high prevalence during adolescence, associated with high morbidity and mortality. In our country, there are no data that characterize adolescent inpatients with ED. Objective: To describe and analyze hospitalizations of children and adolescents due to ED admitted in a Pediatric Mental Health Service (PMHS). Patients and Method: Data were collected from the clinical record of patients with ED hospitalized in the PMHS of the Hospital Roberto del Río during 2005-2015. The following admission variables were studied: cause for hospitalization, ED type, nutritional status, systemic involvement, and psychosocial variables (psychiatric comorbidities, family functioning, abuse, and suicide ideation/attempt). The t-Student test was used for quantitative variables and the chi-square or Fisher Test for qualitative variables for the comparison between groups. Results: 93 patients were included, with an average age of 14.6 years, $84 \%$ of them were women. The most frequent diagnosis was anorexia nervosa (AN) (71\%) and the most frequent cause for hospitalization was the failure of outpatient treatment, followed by suicide ideation/attempt. At admission, $40 \%$ of the patients had malnutrition, $96 \%$ psychiatric comorbidity, and $88 \%$ family dysfunction. Conclusion: AN was the most frequent ED among inpatients and the failure of outpatient treatment was the main cause for hospitalization. The latter could be explained, in part, by the high prevalence of family dysfunction and psychiatric comorbidity of patients and their families which would complicate outpatient treatment.
\end{abstract}

Keywords:

Eeating disorders; anorexia nervosa; adolescent inpatients; family functioning

\section{Introducción}

Los trastornos de la alimentación y de la ingesta de alimentos (TAI) son un grupo de enfermedades psiquiátricas complejas y de difícil tratamiento ${ }^{1,2}$. El DSM-5 reconoce 7 categorías diagnósticas: la anorexia nerviosa (AN), bulimia nerviosa (BN), trastornos por atracones (TA), los trastornos no especificados de la conducta alimentaria (TANE), pica, trastorno de rumiación y trastorno de evitación/restricción de la ingestión de alimentos ${ }^{3}$.

Se estima que la prevalencia internacional de los TAI es de 0,5 a $1 \%$ de la población general. El $85 \%$ de los pacientes desarrolla la enfermedad entre los 13 y 20 años, con un máximo de incidencia entre los 14 y los 16 años $^{4,5}$, siendo más frecuente en mujeres que en

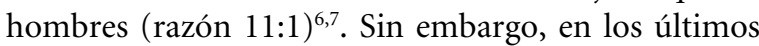
años se ha visto un cambio en el perfil de estos pacientes, la frecuencia de hombres con esta patología ha aumentado y actualmente la razón es de 5:1 y abarca a adolescentes de diferentes niveles socioeconómicos, no sólo del estrato alto ${ }^{8,9}$.

La importancia de estas patologías radica en que presentan una significativa morbimortalidad asociada, cuyo pronóstico mejora con un diagnóstico precoz y tratamiento oportuno. La AN tiene una mortalidad de alrededor de $5,1 \%$ y de está un tercio se explica por suicidio, seguida de trastornos metabólicos e infecciones ${ }^{10}$. En cuanto a la BN, ésta tiene una mortalidad inferior al $3 \%$, con una tasa de complicaciones similar a la observada en $\mathrm{AN}^{11}$.

Por lo anterior, algunas veces no es suficiente el tratamiento ambulatorio y se requiere de la hospitali- zación para su manejo. Las principales causas de hospitalización en TAI son el fracaso del tratamiento ambulatorio y la desnutrición. Sin embargo, la depresión y la ideación suicida han aumentado como razones para el manejo hospitalizado de estas patologías ${ }^{12}$.

La Agencia de Investigación y Calidad de la Salud de EE. UU publicó la estimación nacional de hospitalizaciones para TAI entre los años 1999-2000 a 20052006 y observaron que han aumentado en todos los grupos de edad, con el mayor incremento en niños menores de 12 años $^{13}$. Otros autores han estimado que un tercio de los pacientes con este diagnóstico serán hospitalizados durante su enfermedad ${ }^{14}$.

En Chile no tenemos suficiente información sobre los pacientes con TAI que han requerido hospitalización, cuál de estos trastornos se hospitaliza más, cuáles son las causas de estas internaciones y las características de estos de estos individuos (comorbilidades, situación familiar, abuso, etc). En este estudio se analizan las características de pacientes hospitalizados en un centro de salud mental pediátrico de Santiago.

\section{Pacientes y Método}

\section{Participantes}

Se estudiaron todos los pacientes menores de 18 años hospitalizados en el Servicio de Salud Mental (SSM) del Hospital Roberto del Río con el diagnostico de TAI: AN (tanto restrictiva y purgativa), BN, TA y TANE, entre los años 2005 y 2015. Los pacientes, con estos diagnósticos, se extrajeron de los registros estadísticos de alta. En el SSM se usa la clasificación es- 
tadística internacional de enfermedades y problemas relacionados con la salud (CIE-10) para codificar los diagnósticos. Los datos fueron recolectados por un becado de tercer año de pediatría.

Para el diagnóstico clínico el SSM utiliza el DSM-5 desde el año 2013 y el DSM-IV con anterioridad. El proceso del diagnóstico se realizó mediante una evaluación integral del equipo (siquiatra, sicólogo, adolescentólogo, terapeuta ocupacional y asistente social), buscando concomitantemente la presencia o no de comorbilidad psiquiátrica. Además, se evalúa el desarrollo de personalidad; el funcionamiento familiar y se solicitan las interconsultas a otras especialidades (nutrición, cardiología, etc,). Se hace un plan personalizado de tratamiento centrado en el paciente y sus necesidades (sicoterapia individual, sicofármacos, terapia ocupacional) y su familia (sistémico familiar, especialmente con la figura materna).

Se excluyeron pacientes con genopatías, cardiopatías congénitas e insuficiencia renal.

\section{Parámetros nutricionales}

Desde el punto de vista nutricional se registró peso $\mathrm{y}$ talla al ingreso y al egreso hospitalario. Se consideró eutrófico un índice de masa corporal (IMC) entre $-0,99$ y $+0,99 \mathrm{DE}$, desnutrido IMC $\leq-2 \mathrm{DE}$, en riesgo desnutrición un IMC entre -1 y - 1,99 DE, obesidad $\geq+2 \mathrm{DE}$ y sobrepeso entre $+1 \mathrm{DE}$ y $+1,99 \mathrm{DE}$ para la edad, de acuerdo con las tablas OMS/FAO ${ }^{15}$.

\section{Otras variables}

Para la determinación de la repercusión sistémica se consideró la alteración de los signos vitales. Se definió hipotensión a una presión arterial sistólica y/o diastólica menor al percentil 5 para la talla según las tablas de American Heart Association (AHA) ${ }^{16}$. Se definió taquicardia una frecuencia cardiaca mayor a 100 latidos por minuto $(\mathrm{lpm})$ y bradicardia menor a 60 lpm.

Del registro siquiátrico se obtuvieron los antecedentes de abuso sexual, ideación/intento de suicidio, hospitalización previa y patología siquiátrica concomitante.

Se definió como falla de tratamiento ambulatorio a todo paciente que continúa bajando de peso pese a control ambulatorio independiente del número de controles, aquel con ideación suicida activa, depresión u otra comorbilidad siquiátrica sin respuesta a tratamiento ambulatorio, persistencia de vómitos y disfunción familiar que entrampa el tratamiento.

Se definió como disfunción familiar a las familia sin conciencia de enfermedad y riesgos del TAI; familia que no cumple con las indicaciones de salud mental y/o nutricionales; madre en rol de "cómplice" del TAI de su hijo(a).

\section{Aspectos Éticos}

El protocolo de esta investigación fue revisado y aprobado por el Comité de Ética del Servicio de Salud Metropolitano Norte y el comité interno del Hospital Roberto del Río.

\section{Análisis estadístico}

Se realizó un análisis exploratorio de los datos, en busca de datos anómalos, mal codificados o duplicados. Los resultados se expresaron como promedio y desviación estándar (DE) o mediana y rango según la distribución de cada variable cuantitativa evaluada en forma gráfica con gráficos de densidad de Kernell y prueba de Shapiro-Wilks. Se analizaron las variables dividiendo los datos según el tipo de TAI (AN vs no AN) y los pacientes con AN en aquellos con y sin depresión. Para realizar comparaciones entre grupos se utilizó el test t-Student y para estudiar asociaciones entre variables categóricas se utilizó el test chi-cuadrado o Test Fisher. Se consideró significativo un valor $p<0,05$. Los análisis estadísticos se realizaron en el programa STATA SE 11.2.

\section{Resultados}

Entre los años 2005 y 2015, se hospitalizaron 95 pacientes con diagnóstico de TAI en el SSM del Hospital Roberto del Río, de éstos se obtuvo el registro clínico de 93 pacientes $(97,8 \%)$.

La mayoría de los pacientes fueron mujeres (84\%), con una razón mujer: hombre de 5,2:1; la mediana de edad al diagnóstico fue de 14,1 años $(8-17,5$ años) y al ingreso de la hospitalización fue de 14,6 años (8,1-17,6 años).

La AN fue el principal diagnóstico de ingreso, con un $71 \%$ de los casos, seguido por BN con $18 \%$ y finalmente TANE con $11 \%$. El motivo más frecuente de hospitalización fue el fracaso del tratamiento ambulatorio seguido por la ideación suicida (tabla 1).

$\mathrm{Al}$ ingreso, el 47\% de los pacientes estaban eutróficos y el $40 \%$ presentaban déficit nutricional $(23,7 \%$ estaban en riesgo de desnutrir y el 17,3\% estaban desnutridos) (tabla 1). Al egreso, se observó mejoría del estado nutricional, pero se produjo un aumento en el número de pacientes con sobrepeso de $8,7 \%$ ( 8 pacientes) a $12,6 \%$ (11 pacientes) (figura 1 ).

En cuanto a los signos vitales el $12 \%$ (11 pacientes) presentó bradicardia y el 17\% (16 pacientes) presión arterial baja.

El $96 \%$ de las pacientes tenían al menos una patología psiquiátrica como comorbilidad. Dentro ellas, el diagnóstico más frecuente fue trastorno de la personalidad con $41 \%$ (38 pacientes), seguido por depresión en el $30 \%$ ( 28 pacientes) (tabla 1 ). 
Tabla 1. Características de los pacientes estudiados y comparación entre aquellos con anorexia nerviosa y con otros trastornos de la alimentación e ingesta (TAI)

\begin{tabular}{|c|c|c|c|c|}
\hline Variable & $\begin{array}{c}\text { Total } \\
(n=93)\end{array}$ & $\begin{array}{c}\text { Anorexia } \\
(n=66)\end{array}$ & $\begin{array}{c}\text { Otros TAl } \\
(n=27)\end{array}$ & Valor $p$ \\
\hline Sexo M / H (\%) & $78(84) / 15(16)$ & $52(78,8) / 14(21,2)$ & $26(96,3) / 1(3,7)$ & $0,04^{*}$ \\
\hline Edad de ingreso & $14,6(8,1-17,6)$ & $14,6(11,2-17,5)$ & $14,6(8,1-17,6)$ & $0,3 * * *$ \\
\hline Edad al diagnóstico & $14,1(8-17,5)$ & $14,1(8-17,5)$ & $13,98(8,1-17,1)$ & $0,2 * * *$ \\
\hline $\begin{array}{l}\text { Motivo hospitalización (\%) } \\
\text { - Fracaso tto. ambulatorio } \\
\text { - Ideación/intento suicida } \\
\text { - Riesgo vital } \\
\text { - Disfunción familiar } \\
\text { - Otros o no descrito }\end{array}$ & $\begin{array}{c}45(48,4) \\
20(21,5) \\
10(10,8) \\
1(1,1) \\
17(18,2)\end{array}$ & $\begin{array}{c}37(56,1) \\
9(13,6) \\
8(12,1) \\
0(0) \\
12(18,2)\end{array}$ & $\begin{array}{c}8(29,6) \\
11(40,7) \\
2(7,4) \\
1(3,7) \\
5(18,5)\end{array}$ & 0,01 ** \\
\hline $\begin{array}{l}\text { E. nutricional al ingreso (\%) } \\
\text { - Eutrófico } \\
\text { - Riesgo de desnutrición } \\
\text { - Desnutrición } \\
\text { - Sobrepeso } \\
\text { - Obesidad }\end{array}$ & $\begin{array}{c}44(47,3) \\
22(23,7) \\
16(17,2) \\
8(8,6) \\
3(3,2)\end{array}$ & $\begin{array}{c}32(48,5) \\
17(25,8) \\
14(21,2) \\
2(3) \\
1(1,5)\end{array}$ & $\begin{array}{l}12(44,4) \\
5(19,2) \\
2(7,7) \\
6(23,1) \\
2(7,6)\end{array}$ & 0,01 * \\
\hline $\begin{array}{l}\text { Pat. siquiat. asociada (\%) } \\
\text { - Depresión } \\
\text { - Trastorno de personalidad } \\
\text { - TDAH }\end{array}$ & $\begin{array}{c}28(30,1) \\
38(40,9) \\
3(3,2)\end{array}$ & $\begin{array}{c}19(28,8) \\
24(36,4) \\
2(3)\end{array}$ & $\begin{array}{c}9(33,3) \\
14(53,9) \\
1(3,7)\end{array}$ & $0,6^{*}$ \\
\hline Abuso/violación (\%) & $20(21,5)$ & $10(15)$ & $10(37)$ & $0,02 *$ \\
\hline Ideación suicida (\%) & $31(33,3)$ & $14(21,2)$ & $17(62,9)$ & 0,01 * \\
\hline Disfunción familiar (\%) & $82(88,2)$ & $57(87,7)$ & $25(92,6)$ & $0,5^{*}$ \\
\hline Pat. siquiat. familiar (\%) & $52(56)$ & $36(55,4)$ & $16(59,3)$ & $0,14^{*}$ \\
\hline
\end{tabular}

M/H: mujer/hombre; tto: tratamiento; E. nutricional: estado nutricional; Pat. siquiat: patología siquiátrica; TDAH: trastorno déficit atención e hiperactividad; ${ }^{*}$ chi-cuadrado; $* *$ Test Fisher; ${ }^{* * *}$ Test Student

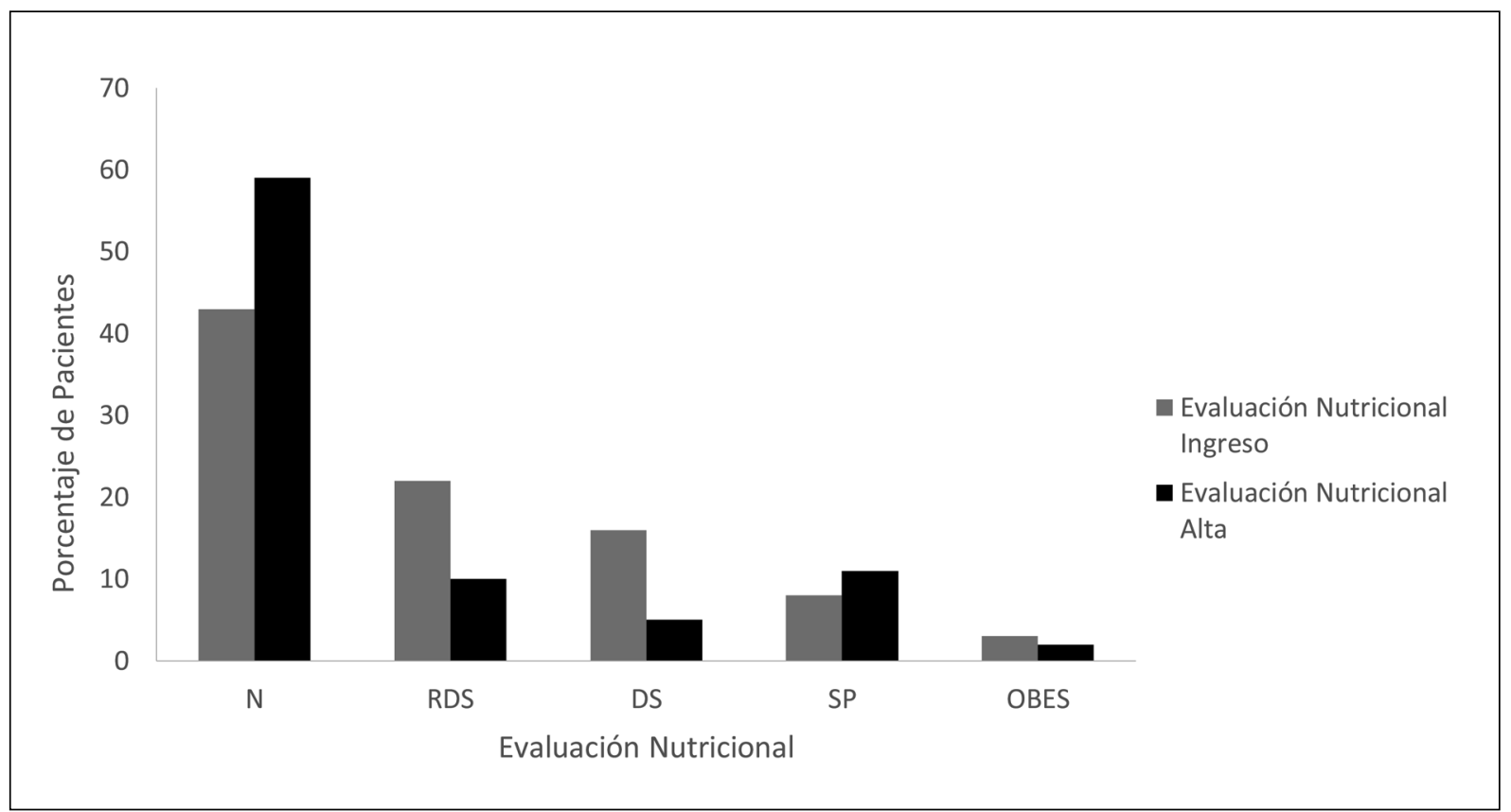

Figura 1. Evaluación nutricional al ingreso y al alta. Se observa al alta un aumento del número de pacientes eutróficos (N) y con sobrepeso (SP) y una disminución de los pacientes con riesgo de desnutrición (RDS), desnutrición (DS) y obesidad (OBES). 
En el análisis de factores sicosociales, destaca la alta prevalencia de disfunción familiar (88\%), seguido de patología siquiátrica familiar (56\%), ideación suicida (33\%) y abuso sexual/violación (20\%). El trastorno de identidad sexual se detectó en 7 pacientes (6 hombres y 1 mujer) de los cuales 6 tenían AN. Del total de pacientes, 17 habían tenido hospitalización previa por TAI $(18,3 \%)$ y de estos 13 tenían AN (tabla 1$)$.

$\mathrm{Al}$ evaluar los periodos de tiempo 2005-2010 y 2010 a 2015, por separado, no se observaron diferencias en el número de pacientes hospitalizados por estas patologías (49 vs 44 adolescentes), sólo hubo diferencias significativas en el motivo de ingreso, donde, aunque el fracaso de tratamiento ambulatorio fue el motivo más común en ambos períodos, en el segundo período aumentaron las hospitalizaciones por ideación/intento suicida y por riesgo vital.

$\mathrm{Al}$ comparar los pacientes con $\mathrm{AN}^{66}$ con aquellos con otros $\mathrm{TAI}^{27}$, destaca que se observó una proporción significativamente mayor de mujeres en los TAI no AN (96\% vs $79 \%)$. Se encontró también diferencia significativa en la causa de hospitalización y el estado nutricional. Por una parte, los pacientes con AN tenían como principal causa de hospitalización el fracaso en el tratamiento ambulatorio, mientras que los otros TAI la ideación/intento suicida. Así mismo, los pacientes con AN tenían mayor compromiso nutricional y menor frecuencia de antecedentes de violación y abuso (tabla1).

$\mathrm{Al}$ analizar dentro del grupo de los pacientes con AN se observó que aquellos que tenían concomitantemente depresión tenían significativamente más ideación/intento suicida $(42,1$ vs $12,8 \mathrm{p}<0,02)$ y aquellos que tenían AN y trastorno de la personalidad tenían mayor disfunción familiar $(100 \%$ vs $76 \% \mathrm{p}<0,01)$.

\section{Discusión}

En esta serie la AN fue el principal diagnóstico de ingreso dentro de los TAI; siendo el fracaso del tratamiento ambulatorio el principal motivo de hospitalización, lo que está de acuerdo a múltiples publicaciones ${ }^{14,17}$. La ideación/intento suicida ocupo el segundo lugar, siendo éste uno de los principales riesgos de estas patologías. Estudios internacionales muestran que los pacientes con TAI tienen 18,1 veces más riesgo de morir por suicidio que pacientes sin este trastorno ${ }^{18,19}$. Sumado a lo anterior, se ha descrito que el número de intentos suicidas están relacionados a mayor duración de la enfermedad ${ }^{20}$.

El antecedente de ideación/intento suicida fue mayor en los TAI no AN, fenómeno ya descrito en publicaciones internacionales, donde se ha observado que los pacientes con $\mathrm{BN}$, por ejemplo, tienen 2,1 veces más ideación/intento suicida que los pacientes con $\mathrm{AN}^{21}$. Se postula que estos eventos son más frecuentes en pacientes con conductas purgativas más que restrictivas $\mathrm{y}$ en aquellos individuos con trastorno de personalidad limítrofe y conductas impulsivas ${ }^{22,23}$, rasgos que se ven más en pacientes con otros TAI no AN.

Como en el resto de los estudios en esta área, se observó predominio de pacientes de sexo femenino, con una relación mujer: hombre de aproximadamente 5:1, lo que es más alto para hombres que lo descrito por Calderón et al $^{14}$ en población norteamericana pero concordante con lo reportado en estudios más recientes, como lo muestra la revisión de Portela et al. ${ }^{24}$. Estas diferencias serían secundarias al incremento de la preocupación por el físico que tienen los adolescentes actualmente?

$\mathrm{Al}$ analizar el estado nutricional de los pacientes, la mayoría de ellos tenían un peso normal, esto explicaría la baja presencia de pacientes con riesgo vital y por otro lado podría sugerir que los pacientes son hospitalizados tempranamente, cuando su tratamiento ambulatorio no está siendo efectivo, evitando las consecuencias orgánicas. El tratamiento precoz de estas pacientes mejora su pronóstico y disminuye las recaídas ${ }^{17,25,26}$. Palazzo et $\mathrm{a}^{27}$ reportaron una posibilidad de remisión del $77 \%$, en pacientes con AN, si el tratamiento es precoz. Sin embargo, si bien se mejoró el estado nutricional de las pacientes en riesgo a desnutrir y desnutridas se vio un aumento de un $4 \%$ de pacientes con sobrepeso al egreso, generalmente dado por pacientes con TAI no AN. Esto debe tenerse en cuenta para mejorar la supervisión nutricional en este aspecto, ya que los pacientes hospitalizados presentan un índice mayor de sedentarismo y los requerimientos calóricos serían menores.

La mayoría de los pacientes tenían otras comorbilidades siquiátricas, lo que se ha visto que aumenta el riesgo a hospitalizaciones y es uno de los predictores de la duración de la estancia hospitalaria ${ }^{28}$. Las comorbilidades más frecuentes fueron el trastorno de la personalidad y la depresión, las que son las más descritas también en la literatura ${ }^{23}$. Cabe notar que la depresión juega un rol muy importante en el futuro del paciente al alta, ya que se ha visto una menor capacidad de mantener el peso en forma ambulatoria ${ }^{29}$. No está claro hasta qué punto estos trastornos del ánimo son secundarios a los trastornos de la alimentación o a condiciones comórbidas secundarias a antecedentes etiológicos compartidos. Por lo anterior, se hace necesario el seguimiento de estos pacientes por periodos largos para poder despejar estas interrogantes.

En cuanto a antecedentes del paciente y la familia, es importante destacar la alta prevalencia, en esta serie, de disfunción familiar, patología siquiátrica en la familia y de abuso sexual ( $90 \%, 56 \%$ y $20 \%$ respectivamente). Varios estudios han reportado una fuerte asocia- 
ción entre experiencias infantiles traumáticas y la gravedad de los síntomas del trastorno alimentario ${ }^{30}$. En especial se ha descubierto que el abuso infantil físico, emocional y/o sexual es un factor de riesgo no específico para el desarrollo de los TAI. Estos factores podrían explicar, en parte, la falla del tratamiento ambulatorio de las pacientes, ya que para la terapia de la paciente se necesita del apoyo y cuidado familiar ${ }^{31,32}$.

Dentro de las limitaciones de este estudio está el hecho que es un trabajo retrospectivo por lo cual los resultados están basados en fichas clínicas y muchas veces no se tienen todos los datos buscados. Por otro parte, no nos permite estudiar si existe causalidad entre los factores estudiados, sólo asociaciones. Muchas veces no hubo claridad en los registros clínicos sobre variaciones de protocolos de alimentación o siquiátricos que pudieran haber cambiado en esas fechas, por lo que no se pudieron tomar como factores a estudiar. Además, durante el periodo de estudio apareció el DSM-5 lo cual podría alterar la clasificación de algunos trastornos. Sin embargo, esta alteración sería leve, ya que los cambios más importantes para este estudio fueron en AN donde se eliminó el criterio de bajo peso (menor a $85 \%$ del peso esperable) y la amenorrea como criterio diagnóstico; estos cambios llevarían más bien a aumentar la prevalencia de $\mathrm{AN}$, ya que se reubicarían las pacientes con TANE que no alcanzaban dichos criterios con el DSM-IV ${ }^{33}$.

A pesar de las limitaciones, éste es el primer estudio que da información descriptiva sobre niños y adolescentes con TAI que han requerido hospitalización en nuestro país.

Los TAI son un problema biopsicosocial que debe abordarse en un primer momento desde la perspectiva de la atención primaria de salud, ya que esta es el área en cual la condición del paciente será detectada primero. La información recopilada en este estudio, si bien sólo demuestran asociaciones, nos da algunas directrices sobre el control de estos pacientes a este nivel. Deberíamos por ejemplo evaluar si existen síntomas de depresión u otras comorbilidades siquiátricas, eventos traumáticos, ideación e intento suicida previo, dinámica familiar y si podemos contar con la colaboración de la familia en el tratamiento; ya que estos factores, como vemos en este estudio, son frecuentes en los pacientes que han requerido hospitalizarse y podrían asociarse, por lo tanto, a una peor evolución ambulatoria. Estos pacientes, debieran tener controles más frecuentes para disminuir o evitar el riesgo de morbimortalidad, hospitalización y duración de esta.

Son necesarios estudios longitudinales en esta área, que nos permitan evaluar cuales son los factores protectores y de riesgo reales para el éxito del tratamiento de los TAI en nuestra población. Por otro lado, en las pacientes hospitalizadas debemos evaluar de alguna manera los protocolos que estamos ocupando, ver su real efectividad de tal manera de tener datos que podamos comparar con otros protocolos y otros centros.

\section{Responsabilidades Éticas}

Protección de personas y animales: Los autores declaran que los procedimientos seguidos se conformaron a las normas éticas del comité de experimentación humana responsable y de acuerdo con la Asociación Médica Mundial y la Declaración de Helsinki.

Confidencialidad de los datos: Los autores declaran que han seguido los protocolos de su centro de trabajo sobre la publicación de datos de pacientes.

\begin{abstract}
Derecho a la privacidad y consentimiento informado: Los autores han obtenido el consentimiento informado de los pacientes y/o sujetos referidos en el artículo. Este documento obra en poder del autor de correspondencia.
\end{abstract}

\section{Conflicto de intereses}

Los autores declaran no tener conflicto de intereses.

\section{Referencias}

1. Rome E.S. Eating disorders in children and adolescents. Curr Probl Pediatr Adolesc Health. 2012;42:28-44.

2. Papadopoulos F, Ekbom A, Brandt L, Ekselius L. Excess mortality, causes of death and prognostic factors in anorexia nervosa. Br J Psychiatry. 2009;194:10-7.

3. Association AP. DSM-5: Diagnostic and Statistical Manual of Mental Disorders. 5th ed. Arlington,VA: American Psychiatric Association; 2013.

4. Marín V. Trastornos de la conducta alimentaria en escolares y adolescentes. Rev Chil Nutr. 2002;29:86-91.

5. Hsu L. Epidemiology of the eating disorders. Psychiatr Clin North Am. 1996; 19:681-700.

6. López C, Treasure J. Trastornos de la Conducta Alimentaria en adolescentes: Descripción y manejo. Rev. Med. Clin. Condes 2011;22:85-97.

7. De La Barra F, Vicente P, Saldivia S, Melipillan S. Estudio de epidemiología psiquiátrica en niños y adolescentes en Chile. Estado actual. Rev Med Clin Condes. 2012; 23: 521-29.
8. Gempeler J. Trastornos de la alimentación en hombres: cuatro subtipos clínicos. Revista Colombiana Psiquiatr. 2006; 35:352-61.

9. Robb A. Eating disorders in children. Diagnosis and age-specific treatment. Psychiatr Clin North Am. 2001; 24:259-70.

10. Campbell K, Peebles R. Eating Disorders in Children and Adolescents: State of the art review. Pediatrics. 2014; 134: 582-92.

11. Arcelus J, Mitchell AJ, Wales J, Nielsen S. Mortality rates in patients with anorexia 
nervosa and other eating disorders: a meta-analysis of 36 studies. Arch Gen Psychiatry. 2011; 68:724-31.

12. Correa M, Zubarew T, Silva P, Romero M. Prevalencia de riesgo de trastornos alimentarios en adolescentes mujeres escolares de la Región Metropolitana. Rev Chil Pediatr. 2006; 77:153-60.

13. Zhao Y, Encinosa W. Hospitalizations for Eating Disorders from 1999 to 2006 HCUP Statistical Brief \#70. April 2009. Agency for Healthcare Research and Quality, Rockville, MD. http://www.hcupus.ahrq.gov/reports/statbriefs/sb70.pdf.

14. Calderon R, Stoep AV, Collet B, Garrison MM, Toth K. Inpatients with eating disorders. Demographic, diagnostic, and service characteristics from a nationwide pediatric sample. Int J Eat Disord. 2007; 40:622-8.

15. WHO Multicentre Growth Reference Study Group. WHO Child Growth Standards: Length/height-for-age, weightfor-age, weight-for-length, weight-forheight and body mass index-for-age: Methods and development. Geneva: World Health Organization, 2006.

16. Chameides L, Samson R, Schexnayder S, Hazinski M, Ashcraft J. Pediatric Advanced Life Support Provider Manual, American Heart Association, 2016.

17. López-de-Andres A, Carrasco-Garrido P, Hernández-Barrera V, Gil-de-Miguel A, Jiménez-Trujillo I, Jiménez-García R. Hospitalization trends in Spanish children and adolescents with eating disorders, (1998-2007). Appetite. 2010;55:147-51.

18. Goldberg S, Werbeloff N, Shelef L, Fruchter E, Weiser M. Risk of suicide among female adolescents with eating disorders: a longitudinal population- based study. Eat Weight Disord. 2015, 20:295-300.

19. Thornton L, Welch E, Munn-Chernoff M, Lichtenstein P, Bulik C. Anorexia Nervosa, Major Depression, and Suicide Attempts: Shared Genetic Factors. Suicide Life Threat Behav. 2016;46:525-34.

20. Tasaka K, Matsubara K, Takamiya S, Ishikawa S, Iwata A, Nigami H. Longterm follow-up for hospitalized children with anorexia nervosa restricting type. Pediatric Int. 2017;59:482-9.

21. Favaro A, Santonastaso P, Monteleone $\mathrm{P}$, et al. Self-injurious behavior and attempted suicide in purging bulimia nervosa: associations with psychiatric comorbidity. J Affect Disord. 2008; 105: 285-9.

22. Mayes S, Fernández J, Bawejaa R, et al. Correlates of Suicide Ideation and Attempts in Children and Adolescents with Eating Disorders. Eat Disord. 2014; 22:352-66.

23. Kaye W, Bulik C, Thornton L, Barbarich N, Masters K. Comorbidity of anxiety disorders with anorexia and bulimia nervosa. Am J Psychiatry. 2004;161:221521.

24. Portela de Santana M, da Costa Ribeiro Junior H, Mora M, Raich R. La epidemiología y los factores de riesgo de los trastornos alimentarios en la adolescencia: una revisión. Nutr Hosp. 2012;27:391-401

25. Treasure J, Stein D, Maguire S. Has the time come for a staging model to map the course of eating disorders from high risk to severe enduring illness? An examination of the evidence. Early Interv Psychiatry. 2015;9:173-84.

26. Treasure J, Russel G. The case for early intervention in anorexia nervosa: theoretical exploration of maintaining factor. Br J Psychiatry. 2011;199:5-7.

27. Palazzo B, Gregor L, Albanol G, et al. Early Response to treatment in Eating Disorders: A Systematic Review and a Diagnostic Test Accuracy Meta-Analysis. Eur Eat Disord Rev. 2017;25:67-79.

28. Strik Lievers L, Curt F, Wallier J, Perdereau F, Rein Z, Jeammet P. Predictive factors of length of inpatient treatment in anorexia nervosa. Eur Child Adolesc Psychiatry. 2009; 18: 75-84.

29. Castro-Fornieles J, Casulà V, Saura B, et al. Predictors of Weight Maintenance after Hospital Discharge in Adolescent Anorexia Nervosa. Int J Eat Disord. 2007; 40:129-35.

30. Palmisano GL, Innamorati M, Susca G, Traetta D, Sarracino D, Vanderlinden J. Childhood Traumatic Experiences and Dissociative Phenomena in Eating Disorders: Level and Association with the Severity of Binge Eating Symptoms. J Trauma Dissociation. 2018;19:88-107.

31. Swenne I, Parling T, Salonen R. Familybased intervention in adolescent restrictive eating disorders: early treatment response and low weight suppression is associated with favourable one-year outcome. BMC Psychiatry. 2017; 17:333.

32. Carter JC, Bewel C, Blackmore E, Woodside DB. The impact of childhood sexual abuse in anorexia nervosa. Child Abuse Negl. 2006;30:257-69.

33. Vázquez R, López X, Ocampo M, Mancilla-Diaz J. Eating disorders diagnostic: from the DSM-IV to DSM5. Revista Mexicana de Trastornos Alimentarios. 2015;6:108-20. 